\title{
DOSE MAPPING OF GAMMA IRRADIATION CHAMBER (GIC)
}

\section{S. Nur Syafinaz ${ }^{1}$, M.R. Mohd Amirul Azrie ${ }^{2}$, A. Nur Amalina ${ }^{2}$ and M.N. Noramaliza ${ }^{2,3^{*}}$}

\author{
${ }^{1}$ Department of Physics, Faculty of Science \\ Universiti Putra Malaysia, 43400 Serdang, Selangor, Malaysia
}
${ }^{2}$ Medical Physics Unit, Teaching Hospital Universiti Putra Malaysia, 43400 Serdang, Selangor, Malaysia

${ }^{3}$ Department of Radiology, Faculty of Medicine and Health Sciences, 43400 Serdang, Selangor, Malaysia

*Corresponding Author's Email: noramaliza@upm.edu.my

Article History: Received January 24, 2022; Revised January 27, 2022;

Accepted January 27, 2022

\begin{abstract}
The use of gamma rays to irradiate blood products have been demonstrated to reduce the probability of post-transfusion graft versus host disease. The aim of this study is to investigate the accuracy of a new batch of Gafchromic EBT-XD films to map the homogeneity of gamma radiation dose delivered to the blood bags and identify the lowest and highest levels of radiation exposure to the blood. The Gammacell 3000 Elan Gamma irradiation chamber (GIC) @ Blood Irradiator with Cesium-137 source was used. During the gamma irradiation at an exposure of 8 minutes 51 seconds to deliver a central dose of 25 Gy to the film, the EBT-XD films were placed at the centre of a blood-equivalent phantom. The doses then were measured using dose mapping analysis of Gafchromic EBT-XD film. The responses of EBT-XD films were compared with EBT3 films and GS 300 Dose Mapping Report 2021 prepared by Best Theratronics Dosimetry Laboratory, Canada. Results showed the value of doses obtained from the mapping using EBT-XD were reliable compared with the doses measured in EBT3 film as they were in the range of the acceptable dose ranges for blood irradiation procedures. The mapping with EBT-XD film yielded a minimum dosage of $18 \mathrm{~Gy}$ and a maximum exposure of $32 \mathrm{~Gy}$ at 8 minutes 51 seconds of irradiation. In conclusion, EBT-XD offered excellent dosimetric characteristics and has a the potential to dose map the blood irradiator.
\end{abstract}


KEYWORDS: Blood irradiator; EBT3 film; EBT-XD film; dosimetry; dose-mapping

\subsection{INTRODUCTION}

A way of transferring blood or blood products, from one person to another after blood is lost through an illness or injury is called a blood transfusion. A transfusion generally can help in supplying the replacement or reinforcement to one or more components that make up the healthy blood if the body is missing any one or more of them. Some of the reasons why people might need to receive a blood transfusion are sickle cell disease, a bleeding disorder such as cancer or haemophilia, and anaemia. Patients who suffered from anaemia or an iron deficiency may require a red blood cell transfusion. Platelets are blood cells that aid in the prevention of bleeding. Platelet transfusion is desired if the human body doesn't have enough platelets. This might happen as a result of cancer or cancer treatments. Blood transfusions are generally considered safe, however, there are complications involved. Some issues appear right away, while others take a while.

Transfusion-associated graft-versus-host disease (TA-GVHD) is an uncommon blood transfusion complication in which immunologically competent donor $\mathrm{T}$ cells initiate an immune response against the lymphoid tissue of the recipient [1]. In order to prevent the TA-GVHD, the standard method that is currently used in clinical practice is the irradiation of cellular blood products using either gamma rays or Xrays. The purpose of the irradiation is to inactivate the T-lymphocytes found in cellular blood products, preventing blast transformation and mitotic activity [2]. By using gamma irradiation either from Cesium137 or Cobalt-60, with a minimum dose of $25 \mathrm{~Gy}$, the effect could be achieved.

Prior to actual irradiations being carried-out, the dose mapping analysis using various type of dosimeters are performed to ensure the gamma radiation dose to the blood products are within the specified limits of a minimum of $25 \mathrm{~Gy}$ and a maximum of $50 \mathrm{~Gy}$ [1]. It is to make sure the given doses are enough to prevent graft versus host disease in even paediatrics and transplant patients. Other than that, the homogeneity of radiation doses on the blood package also need to be performed to ensure the whole blood package received the same exposure regardless at the centre of the package or at the periphery.

Film dosimeter is one of the dosimeters that is oftenly used to map dose in blood irradiator e.g. EBT3 and EBT-XD types. EBT3 film is 
made up of a single active layer that contains the active substance, a marker dye, stabilisers, and other additives, resulting in a film with a low energy dependence. The images are acquired with flatbed scanners, EBT3 features a unique polyester substrate that inhibits the development of Newton's Rings interference patterns. While for EBT$\mathrm{XD}$ film in accordance to the product specification notes, it has one active layer, with $25 \mu \mathrm{m}$ thick. The active layer is symmetrically sandwiched between two $125 \mu \mathrm{m}$ thick matte polyester substrates. The active layer and the polyester substrates in the EBT-XD film are thinner than those in the EBT3 film (active layer: $28 \mu \mathrm{m}$; polyester substrate: $125 \mu \mathrm{m})$ [3].

This study aimed to investigate the uniformity of the dosage of radiation delivered to the blood and to establish the minimum and maximum radiation doses that the blood has been exposed to by using EBT3 and EBT-XD films.

\subsection{METHODOLOGY}

\subsection{Samples preparation, irradiations and dosimetry}

In this study, a Gammacell 3000@ Elan Blood Irradiator with Cesium137 source located at Department of Pathology, Universiti Putra Malaysia was used. The absorbed dose distributions were measured using EBT-XD (Lot \#: 08021)- and EBT3 (Lot \#: 03071601) radiochromic films (Ashland, US). A blood-equivalent phantom manufactured by Best Theratronics Dosimetry Laboratory, Canada was positioned in the blood canister prior to the irradiation of the films.

\subsubsection{Film dose calibration}

A total of eight pieces of EBT3 and EBT-XD films were used to obtain dose calibration curves. Prior to irradiation, the films were cut into 11.7 $\mathrm{cm} \times 19.3 \mathrm{~cm}$ and positioned at the centre of the blood-equivalent phantom. Seven pieces of the films were then exposed to gamma-ray with doses ranging from 5 up to $35 \mathrm{~Gy}$, while one film was reserved as a control sample and left unexposed. The dose calibration curves of the films (net OD versus dose) were plotted using three channels (red, green and blue) scan modes in order to choose the best channel to obtain the optimum curves for the films.

\subsubsection{Dose Homogeneity}

For the dose homogeneity measurements, the films were irradiated at the centre of a blood-equivalent phantom after being positioned in the 
blood canister. The irradiation exposure of 8 minutes 51 seconds was used to deliver a central dose of $25 \mathrm{~Gy}$ to the film. The number of grids used for mapping was based on the GS Dose Mapping Report 2021 (12 columns, 20 rows). The mapping was done using Origin software for contouring.

\subsection{Scanning Protocol and Analysis}

All the irradiated films used in this study were scanned after 48 hours post-irradiation using a Microtek ScanMaker 1000 XL (Taiwan) flatbed scanner. The transmission mode was set according to the set-up by $[3,4]$ at the scanner including scan resolution of 75 dots per inch (dpi), a 48bit TIFF image ( 16 bits per channel) and all available image correction methods turned off. All film pieces were positioned at the same location in the centre of the scanning area. A $41 \mathrm{~cm} \times 36.1 \mathrm{~cm}$ cardboard template was affixed to the scanner to placed films in a repeatable and consistent centre spot on the scan area.

The template was also used to reduce the effect of lateral dependency artefacts (nonuniform readout response induced by light scattering from particles in the film active layer). Five OD measurements were taken across the central region of the scanner to validate this assumption, with a standard error of less than 0.06 per cent.

Distinctive channel values were extracted by using ImageJ v1.49. A region of interest $(\mathrm{ROI})$ of $1.0 \mathrm{~cm} \times 1.0 \mathrm{~cm}$ region at the centre of each film was selected. The net OD was calculated as shown in Equation 2.1 [4]:

$$
n e t O D=O D_{\text {exposed }}-O D_{\text {unexposed }}=\log _{10} \frac{I_{\text {unexposed }}}{I_{\text {exposed }}}
$$

where Iunexposed and Iexposed are the readings for unexposed and exposed film pieces, respectively.

\subsection{RESULTS AND DISCUSSION}

\subsection{Film Dose calibration}

The calibration curves obtained for the red, green and blue channels over a dose range from 0 to $35 \mathrm{~Gy}$ are shown in Figures 1 and 2. The Rsquare values, which assess the goodness of the fit, were very close to 
unity. Based on Figure 1, as the dose increased, the optical density increased. The growth of the EBT3 red channel was particularly sharp up to around $10 \mathrm{~Gy}$, when the sensitivity was quite strong. The optical density gradually increased at greater doses until it reached saturation. For higher levels of doses, the green channel outperformed the red, indicating that using the green channel at larger doses may be preferable. The green channel showed very high sensitivity and low uncertainty after $25 \mathrm{~Gy}$. Due to the influence of the yellow marker dye, the blue channel of both film types had a smaller response gradient at any dosage. The signal in the blue channel was very slightly affected by the active layer thickness, but the signal in the red channel was significantly affected. Thus, it seemed that the blue channel was less valuable for dose measurement than the green and red channels. Several studies have been published that utilised the blue channel as an optimum analytical approach in triple-channel dosimetry $[5,6,7]$.

For doses more than 5 Gy, EBT-XD films outperformed EBT3 in terms of sensitivity as shown in Figure 2. At doses larger than $10 \mathrm{~Gy}$, the slopes of the response function for the EBT-XD film had a broader dynamic range than those for the EBT3. The red channel of the EBT-XD film was utilized in this research study as it had a wider dynamic range than the EBT3 film. The signal of this channel was much higher than the others.

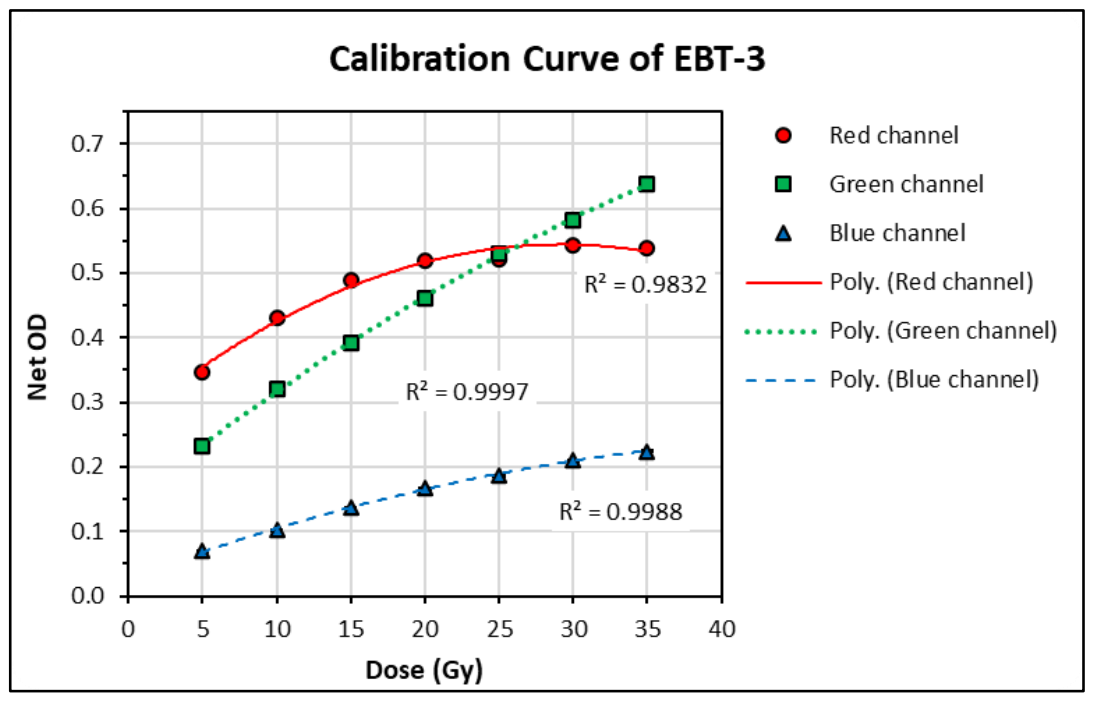

Figure 1: Calibration curve of EBT3 for the red, green and blue channel 


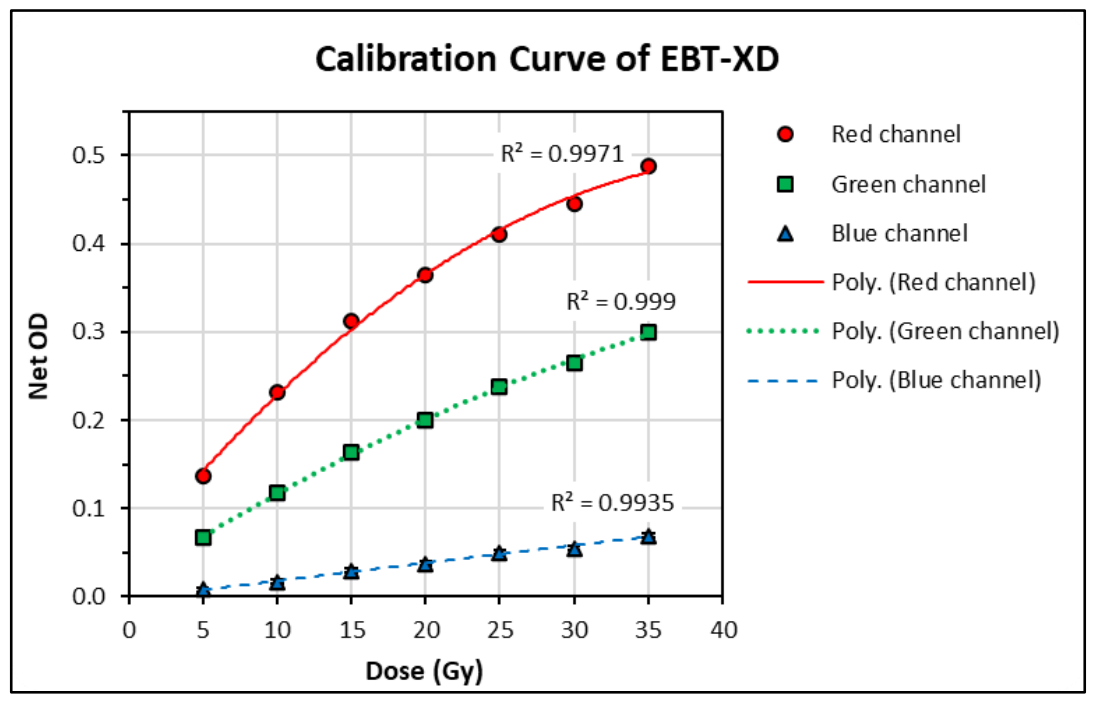

Figure 2: Calibration curve of EBT-XD for the red, green and blue channel

\subsection{Dose Homogeneity}

The results of the determination of the dose for mapping using the EBT3 and EBT-XD film are shown in Figures 3 and 4. Figure 5 illustrates the dose mapping using radiochromic film from Report 2021.

\subsubsection{EBT3 Film}

The doses at the peripheral areas of the EBT3 films were greater than $15 \mathrm{~Gy}$ as illustrated in Figure 3. However, some area of the central region of the film was less than $25 \mathrm{~Gy}$. These values did not reach the minimum dose required at the centre to eliminate the proliferative of lymphocytes. The minimum central dose required to inactivate the Tlymphocytes was 24 Gy where a measured value of 2400 cGy might range from 2520 cGy to 2380 cGy [2]. Therefore, the use of Gafchromic EBT3 film for dose-mapping of the blood irradiator was not recommended as it was inaccurate.

\subsubsection{EBT-XD film}

On the other hand, the doses in the peripheral areas of EBT-XD film were greater than $17 \mathrm{~Gy}$. The doses at the central region of the film were between 24 Gy to 25 Gy as shown in Figure 4. The minimum dose obtained was 18 Gy while the maximum dose was 32 Gy. A similar conclusion was reached by Olivo et al 2015 [1] who performed a study on blood irradiation using a linear accelerator. They believed that the 
accepted dose range to kill the T-lymphocytes was between 15 Gy to 50 Gy. Hence, Gafchromic EBT-XD film was the most suitable type of film to be used for dosimetry of the blood irradiator. In addition, the pattern of dose mapping for EBT-XD film matched with the Report 2021 displayed in Figure 5. The doses obtained from mapping using both EBT3 and EBT-XD films were in agreement with that obtained from the use of the radiochromic film to within $\pm 8 \%$ and $\pm 6 \%$ respectively.

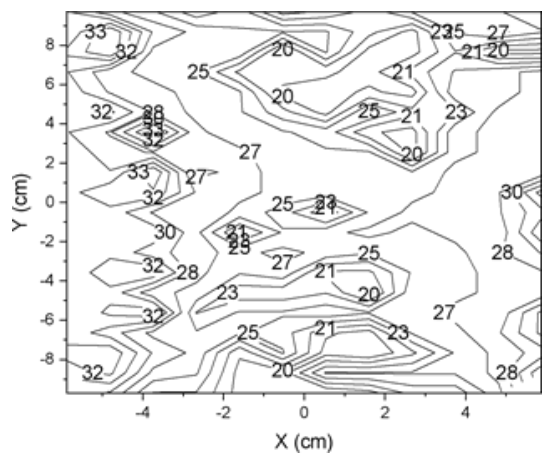

(a)

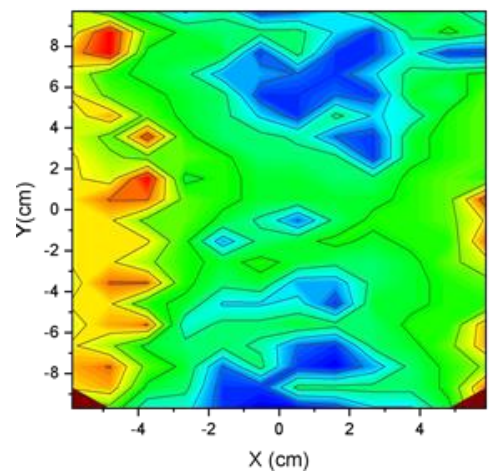

(b)

Dose (Gy)

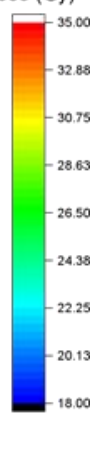

Figure 3: Dose-mapping of a Gammacell 3000 Elan Blood Irradiator using Gafchromic EBT3 film. (a) Contour black and white (b) Contour colour fill

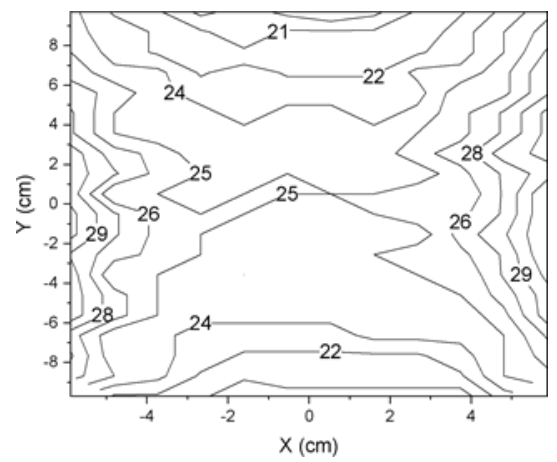

(a)

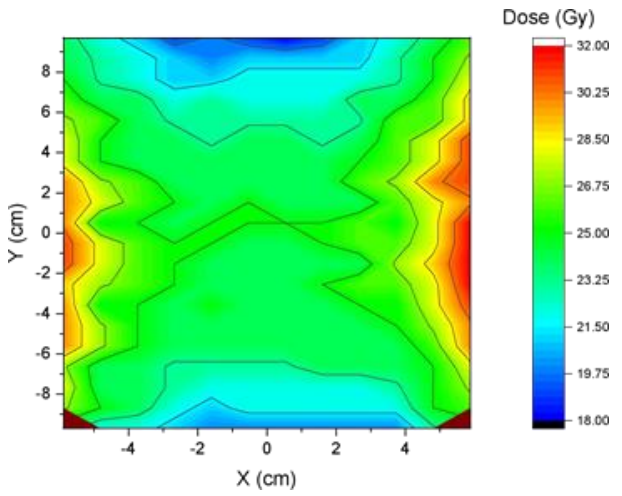

(b)

Figure 4: Dose-mapping of a Gammacell 3000 Elan Blood Irradiator using Gafchromic EBT-XD film. (a) Contour black and white (b)

Contour colour fill 


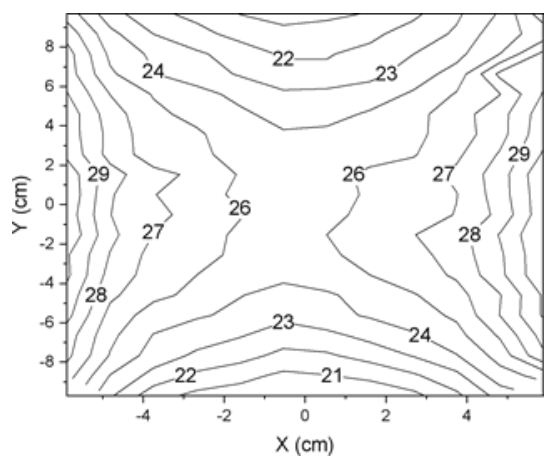

(a)

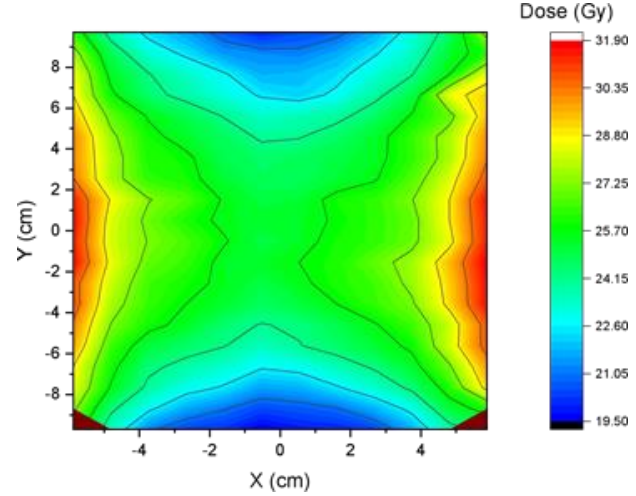

(b)

Figure 5: Dose-mapping of a Gammacell 3000 Elan Blood Irradiator using radiochromic film from Report 2021.(a) Contour black and white (b) Contour color fill

\subsection{CONCLUSION}

The doses in the peripheral areas were observed to be lesser than the central area, for both types of films. EBT-XD film was the most appropriate dosimeter for dose verification compared to EBT3 film. This is due to the fact that for dose-mapping using EBT-XD film, the doses obtained on the film were in the range of doses needed to inhibit the proliferation of the T-lymphocytes as mentioned in [1]. The minimum and maximum dose was 18 Gy and 32 Gy respectively.

\subsection{ACKNOWLEDGEMENTS}

The authors would like to thank the staff and medical physicists from the Radiology and Pathology Department of Universiti Putra Malaysia Teaching Hospital (HPUPM) for their help in completing this study. The authors also appreciate National Cancer Institute for their help with the scanning of the films.

\subsection{REFERENCES}

[1] Olivo, R. A., Silva, M. V. D., Garcia, F. B., Soares, S., Rodrigues, V., \& Moraes-Souza, H. (2015). Evaluation of the effectiveness of packed red blood cell irradiation by a linear accelerator. Revista brasileira de hematologia e hemoterapia, 37, 153-159.

[2] Saglam, S., Cakir, A., \& Kuter, S. (2011). Blood irradiation. In 
Modern Approaches To Quality Control. IntechOpen.

[3] Casolaro, P. (2021). Radiochromic films for the two-dimensional dose distribution assessment Applied Sciences, 11(5), 2132.

[4] Dreindl, R., Georg, D., \& Stock, M. (2014). Radiochromic film dosimetry: considerations on precision and accuracy for EBT2 and EBT3 type films. Zeitschrift für Medizinische Physik, 24(2), 153163.

[5] Miura, H., Ozawa, S., Hosono, F., Sumida, N., Okazue, T., Yamada, K., \& Nagata, Y. (2016). Gafchromic EBT-XD film: dosimetry characterization in high-dose, volumetric-modulated arc therapy. Journal of Applied Clinical Medical Physics 17(6), 312322.

[6] Mohammadyari, P., Zehtabian, M., Sina, S., Tavasoli, A. R., \& Faghihi, R. (2014). Dosimetry of gamma chamber blood irradiator using PAGAT gel dosimeter and Monte Carlo simulations. Journal of Applied Clinical Medical Physics, 15(1), 317-330.

[7] Palmer, A. L., Dimitriadis, A., Nisbet, A., \& Clark, C. H. (2015). Evaluation of Gafchromic EBT-XD film, with comparison to EBT3 film, and application in high dose radiotherapy verification. Physics in Medicine \& Biology, 60(22), 8741. 